\title{
Equating stimulus intensities by means of the GSR'
}

The purpose of this study was to match intensities of stimuli of different sense modalities on the bas is of the magnitude of GSR. Twenty-five college students received repeated presentations of each of 6 different intensities of a 1,000 cps tone $(43,48,53,58,63$, and $68 \mathrm{db}$ re: .0002 dynes $/ \mathrm{cm}^{2}$, in $42 \mathrm{~dB}$ of background noise) alternating with a $1 / 4$ in. square patch of white light of $0.36 \mathrm{ft} .-\mathrm{c}$. Average GSR magnitude was determined. for each of these stimuli. A graph of the relationship between tone intensity and magnitude of GSR was plotted separately for each $S$ and a tone intensity equivalent to the light was determined by interpolating the average maynitude of response to the light in this graph.

The purpose of this study was to match weak visual and auditory stimuli on the basis of the magnitude of the GSR they elicited. Stevens, Mack, \& Stevens (1960) have pointed out that their use of a hand dynamometer in an intermodal matching situation relieved the $S$ of the burden of making numerical estimations of intensity and, thus, reduced error from that source. The present study required of the $S$ merely that he pay attention to the stimuli. Intensity estimation was eliminated in this way and the $S$ became a passive intensity-registering "instrument."

The auditory stimuli used were close to the absolute intensity threshold and were varied in steps of $5 \mathrm{~dB}$ (re: .0002 dynes $/ \mathrm{cm}^{2}$ ). For this reason the study also provided a useful extension of the Hovland \& Riesen (1940) findings on GSR and tone intensity, since they sampled a wide range of intensities but used only one intensity near the theshold.

\section{Subjects and Instructions}

Twenty-five Florida undergraduate psychology students were Ss. They were either paid $\$ 1.00$ for serving or did so to meet a class requirement. All instructions were read to $\mathrm{S}$. They told him to relax and pay attention to the stimuli and to avoid unnecessary movements.

\section{Apparafus}

The duration of both stimuli was 1.0 sec., controlled electronically. The auditory stimulus was a $1000 \mathrm{cps}$ tone produced and amplified by General Radio Company equipment. $E$ varied the intensity of the tone from trial to trial by manipulating an attenuator. The tone was delivered via a single earphone, Type ANB-H-1, suspended $30 \mathrm{in}$. in front of S's head, behind a black cloth. Tone intensity was measured by a sound pressure level meter, approximately 6 in. in front of the earphone. The six measured intensities used were $43,48,53,58,63$, and $68 \mathrm{~dB}$ (re: .0002 dynes/ $\mathrm{cm}^{2}$ ).

An air conditioning fan which was on during the experiment registered $42 \mathrm{~dB}$ on the meter. Data collected by Hawkins \& Stevens (1950) indicate that the absolute threshold for a $1000 \mathrm{cps}$ tone is raised to approximately $60 \mathrm{~dB}$ by a masking white noise at the level of the present air conditioning fan, so that the three lowest intensities were barely, if at all, audible.

The visual stimulus was produced by a $35 \mathrm{~mm}$ projector enclosed in the panel which housed the sound source. The light was passed through a $1 / 4-$ in. square opening $10 \mathrm{in}$. under the earphone. The light's intensity, measured by a MacBeth illuminometer placed directly in front of the square opening, averaged 0.36 $\mathrm{ft}$ - $\mathrm{c}$. over 10 readings.

The palmar GSR was picked up as a DC resistance change by $3 / 4-$ in. zinc electrodes in lucite cups filled with saline jelly (Grings, 1954). It was amplified by a Hunter GSR amplifier and recorded on an EsterlineAngus ink-writing milliameter with a paper speed of $3 \mathrm{in.} / \mathrm{min}$. The GSR unit used was the square root of conductance change, measured within a 3-sec. interval beginning $1.0 \mathrm{sec}$. after stimulus onset.

\section{Procedure}

The $S$ sat in a cushioned chair in a sound-proof room lighted only by a shielded $7.5-\mathrm{w}$ red bulb. The instructions were read and the electrodes attached. The doors were then closed and the stimulus presentation begun.

The method of constant stimuli was used to deliver the six intensities, with the light presented alternately between tones. A random sequence of tone intensities was used, except that the $68 \mathrm{~dB}$ intensity was delivered on the first trial. Time between stimuli was varied unsystematically between 10 and $30 \mathrm{sec}$.

Each intensity was delivered 10 times, and the light was delivered 60 times. Ss who habituated to the stimuli to the extent of failing to respond to $75 \%$ of the last 20 trials in the first 60 , were terminated at that point. There were $10 \mathrm{Ss}$ in this group. The remaining 15 Ss received the entire 120 presentations. Results and Discussion

Average GSR magnitude was determined for each intensity of the tone and for the light, separately for each S. For the $10 \mathrm{Ss}$ who had received only 60 trials, these means are based on five values for each tone 
Table 1. Average GSRs to Light and Tones

\begin{tabular}{|c|c|c|c|c|c|c|c|c|}
\hline & & & & $\ln t e$ & $1 T y$ & Ton & & \\
\hline Subject & +43 & 48 & 53 & 58 & 63 & 68 & Light & $\begin{array}{l}\text { Intensity of } \\
\text { Matching Tone (db) }\end{array}$ \\
\hline 1 & 0.38 & 0.54 & 0.23 & 0.18 & 0.70 & 1.13 & 0.47 & 58 \\
\hline 2 & 0.33 & 0.67 & 0.63 & 0.90 & 1.15 & 1.42 & 0.55 & 52 \\
\hline 3 & 0.25 & 0.20 & 0.23 & 0.20 & 0.34 & 0.74 & 0.33 & 61 \\
\hline 4 & 0.18 & 0.14 & 0.16 & 0.13 & 0.24 & 0.45 & 0.21 & 62 \\
\hline 5 & 0.20 & 0.32 & 0.48 & 1.30 & 0.71 & 0.67 & 0.47 & 52 \\
\hline 6 & 0.39 & 0.18 & 0.27 & 0.36 & 0.44 & 1.22 & 0.24 & 51 \\
\hline 7 & 1.21 & 1.15 & 1.16 & 1.34 & 1.63 & 2.21 & 1.16 & 53 \\
\hline 8 & 0.48 & 0.68 & 0.60 & 0.82 & 1.50 & 3.11 & 0.92 & 59 \\
\hline 9 & 0 & 0 & 0 & 0 & 0.04 & 0.22 & 0.03 & 61 \\
\hline 10 & 0.95 & 0.17 & 0.51 & 0.18 & 0.90 & 1.80 & 0.50 & 60 \\
\hline 11 & 0.10 & 0.26 & 0.13 & 0.32 & 0.49 & 0.87 & 0.37 & 61 \\
\hline 12 & 0.14 & 0.18 & 0.08 & 0.46 & 0.63 & 1.08 & 0.34 & 57 \\
\hline 13 & 0.28 & 0.19 & 0.29 & 0.31 & 0.44 & 0.55 & 0.33 & 58 \\
\hline 14 & 0.67 & 1.12 & 0.87 & 0.30 & 1.96 & 2.20 & 0.64 & 47 \\
\hline 15 & 0.03 & 0.22 & 0.07 & 0.18 & 0.04 & 0.57 & 0.04 & 50 \\
\hline 16 & 0 & 0.12 & 0 & 0 & 0.21 & 0.75 & 0.07 & 57 \\
\hline 17 & 0.03 & 0.08 & 0 & 0.20 & 0.38 & 0.24 & 0.12 & 60 \\
\hline 18 & 0.16 & 0.09 & 0 & 0.11 & 0.02 & 0.19 & 0.12 & 66 \\
\hline 19 & 0.23 & 0.40 & 0.13 & 0.36 & 0 & 1.08 & 0.41 & 61 \\
\hline 20 & 0 & 0.08 & 0 & 0 & 0.06 & 0.80 & 0.09 & 61 \\
\hline 21 & 0.46 & 0.09 & 0.17 & 0.23 & 0.67 & 0.55 & 0.44 & 62 \\
\hline 22 & 0.55 & 0.55 & 0.31 & 0.50 & 0.67 & 1.61 & 0.83 & 64 \\
\hline 23 & 1.75 & 1.01 & 1.24 & 2.04 & 2.89 & 3.89 & 2.69 & 62 \\
\hline 24 & 0.07 & 0.24 & 0.10 & 0.16 & 0.58 & 1.22 & 0.59 & 63 \\
\hline 25 & 0.11 & 0.48 & 0.50 & 0.95 & 0.78 & 0.98 & 0.46 & 58 \\
\hline Mean & 0.36 & 0.37 & 0.33 & 0.46 & 0.70 & 1.18 & 0.50 & 58.2 \\
\hline SD & 0.41 & 0.33 & 0.34 & 0.49 & 0.67 & 0.82 & 0.52 & - \\
\hline
\end{tabular}

and 30 for the light; for the remaining $15 \mathrm{Ss}$, the means are based upon 10 values for each tone and 60 for the light. Table 1 presents these means for all $25 \mathrm{Ss}$, and indicates the total means and standard deviations for the light and tones.

A smoothed curve of the relationship between tone intensity and response magnitude was estimated visually for each $\mathrm{S}$. The value of the mean magnitude of response to the light was then located on the $Y$ axis of this graph and the corresponding tone intensity determined. These intensities are presented in the last column of Table 1 .

Most of the $25 \mathrm{Ss}$ showed the same type of variation in GSR magnitude as a function of stimulus intensity, as is indicated in Table 1. Because of the regular pattern of variation in the GSR means as a function of stimulus intensity, it was of interest to represent this relationship graphically, as is done in Fig. 1.

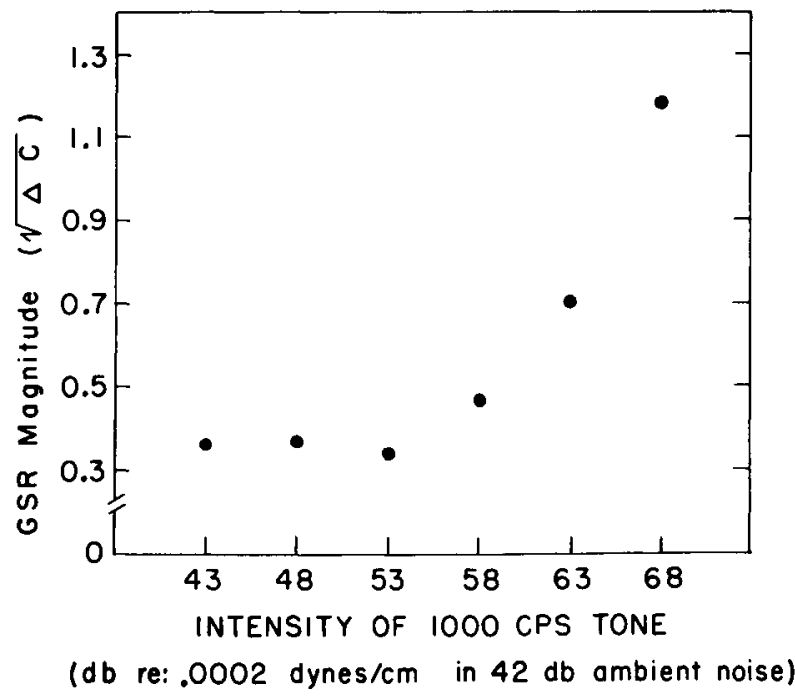

Fig. 1. Magnitude of galvanic skin response as a function of of intensity of a 1,000 cps pure tone, presented in $42 \mathrm{db}$ of background noise.

As can be seen in the figure, as tone intensity was increased in the threshold region, a positively accelerated function was described.

Hovland \& Riesen (1940) have shown that a slight amount of negative acceleration in the GSR intensity curve can be expected in the upper intensity region, indicating, in conjunction with the present data, that an S-shaped function would probably be obtained if several near-threshold values were included.

\section{References}

Grings, W. W. Laboratory instrumentation in psychology. Palo Alto: National Book Company, 1954.

Hawkins, J. E., \& Stevens, S. S. The masking of pure tones and of speech by white noise. J. Acoust. Soc. Amer., 1950, 22, 6-13. Hovland, C. I., \& Riesen, A. H. Magnitude of galvanic and vasomotor response as a function of stimulus intensity. J. gen. Psychol., 1940, 23, 103-121.

Stevens, J. C., Mack, J. D., \& Stevens, S. S. Growth of sensation on seven continua as measured by force of hand-grip. $J$. exp. Psychol., 1960, 59, 60-67.

\section{Note}

1. The work described in this report was done at the University of Florida under contract with the Personnel and Training Branch of the Office of Naval Research (NONR $580(09)$ ). 